\title{
PERFIL DE LAS TESIS PARA LA OBTENCIÓN DEL TÍTULO PROFESIONAL EN LA ESCUELA NACIONAL SUPERIOR DE FOLKLORE JOSÉ MARÍA ARGUEDAS
}

\author{
Xavier FUENTES AVILA \\ Escuela Nacional Superior de Folklore José María Arguedas \\ xfuentesavila@gmail.com \\ Miriam CARREÑO COLCHADO \\ Universidad Nacional Mayor de San Marcos \\ miriamcarreno@gmail.com
}

\section{RESUMEN}

En el presente trabajo se realizó un análisis bibliométrico de las tesis presentadas para obtener el título profesional en la Escuela Nacional Superior de Folklore José María Arguedas (La Escuela) y que se encuentran a disposición en la biblioteca de dicha institución.

Se analizaron 77 tesis de un total de 163, con los objetivos de cuantificar la producción de trabajos de investigación en La Escuela, realizar una caracterización temática, analizar la fundamentación teórica, revisar la metodología y realizar una prospección sobre la temática de las tesis.

Este estudio ha permitido conocer las tendencias en cuanto a las temáticas de las tesis y la predilección que muestran los alumnos por trabajar con hechos folklóricos de la costa y sierra de nuestro país. No se ha encontrado ninguna tesis que trabaje manifestaciones folklóricas de la región natural selva.

Se ha encontrado, además, que los temas relacionados a la Educación Básica Regular son los que tienen una mayor atención; que los temas dedicados a las danzas superan visiblemente a los de música y que el número de tesis sustentadas ha tenido una notable variabilidad lo que no permite establecer una tendencia en la producción de las tesis por año.

\section{PALABRAS CLAVE}

Tesis, folklore, educación, música, danza.

\section{PROFILE OF THE THESIS TO OBTAIN THE PROFESSIONAL DEGREE IN THE ESCUELA NACIONAL SUPERIOR DE FOLKLORE JOSÉ MARÍA ARGUEDAS}

\section{ABSTRACT}

In the present paper, a bibliometric analysis of the theses presented to obtain the professional degree in the Escuela Nacional de Folklore José María Arguedas (La Escuela) which are available in the library of said institution was carried out.

77 theses of a total of 163 were analyzed, in order to quantify the production of research papers at "La Escuela", conducting a thematic characterization, analyzing the theoretical foundation, reviewing the methodology and conducting a survey on the thematic of the thesis. 
This study has allowed to observe the tendencies regarding the themes of the theses and the predilection that students show for working with folkloric facts of the coastal and mountain regions of our country. No thesis has been found that focuses on folkloric manifestations of the jungle natural region.

It has also been found that the issues related to Regular Basic Education are those that have the most attention, that the themes dedicated to dances visibly exceed those of music and that the number of theses supported has had a remarkable variability which does not allow for the establishment of a trend in the production of theses per year.

\section{KEY WORDS}

Thesis, folklore, education, music, dance.

Recibido: $13 / 03 / 2019$

Aprobado: 10/05/2019

\section{INTRODUCCIÓN}

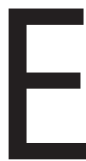

n el marco de la investigación folklorológica y pedagógica en el Perú, por diversas razones la danza y música folklóricas, así como la didáctica de su enseñanza, son entidades que han recibido un escaso tratamiento como objetos de estudio científico ${ }^{1}$. Esto significa que los estudiantes de la Escuela Nacional Superior de Folklore José María Arguedas (La Escuela) cuentan con antecedentes relativamente escasos para guiar la elaboración de la tesis de licenciatura.

La exigencia de elaborar una tesis es un requisito que ha permanecido constante durante toda la existencia de La Escuela. La forma y el contenido de esos trabajos han presentado variaciones características de ciertas épocas.

En 1949, mediante el Decreto Supremo № 1053 se autoriza el funcionamiento de la denominada Escuela de Música y Danzas Folklóricas Peruanas. En esta, su primera etapa de existencia oficial que se prolonga hasta 1964, las exigencias para la obtención del título profesional incluyeron, según lo señala Arana (2007), "presentar un trabajo sobre folclor" (p.32), trabajo que, independientemente del grado de dificultad que comporte su realización, constituye una investigación. Todos ellos se encuentran catalogados y almacenados en la biblioteca de la institución, con la denominación de tesis².

Una segunda etapa, se produce en abril de 1965, cuando mediante Resolución Suprema No 504 se dispone que la entidad pase a la jurisdicción del Ministerio de Educación. Este cambio produce en toda la institución, una importante impregnación del carácter pedagógico, propio de las entidades dedicadas a la formación docente

\footnotetext{
1 Considérese que la investigación en arte o en historia del arte privilegia el tratamiento de las artes plásticas, tal como lo evidencian los currícula universitarios y las diversas publicaciones sobre la materia.

2 Para el presente estudio, se consideran como pertenecientes a esta etapa, las tesis producidas entre 1957 y 1963.
} 
dependientes del Ministerio de Educación y que se prolongará solo hasta 1969. A partir de ese año la formación docente se suspende durante dos décadas. Pese a ello, se han encontrado en biblioteca tesis con fecha de sustentación entre 1971 y $1974^{3}$.

En 1989 se inicia una tercera etapa con la entrada en vigencia del Decreto Supremo № 02-89-ED que pone en funcionamiento La Escuela Nacional Superior de Folklore José María Arguedas, con facultad para expedir título profesional de Profesor de Educación Artística en la especialidad de Folklore, con mención en Música o Danza. Esta etapa se prolonga hasta el año 2008. La primera tesis correspondiente a este período data del año 1994 y todas las demás hasta la última que obra en poder de la biblioteca -sustentada el 2017-, pertenecen al mismo período. ${ }^{4}$

Se puede identificar una cuarta etapa, a partir de la publicación de la Ley 29292 en diciembre del 2008, norma en la que se faculta a La Escuela José María Arguedas para otorgar grado de bachiller y título de licenciado, equivalentes a los otorgados por las universidades. No obstante que La Escuela tiene nueve años de recibir estudiantes dentro del sistema universitario, hasta la fecha de culminación del presente estudio, no se ha encontrado en la biblioteca, ninguna tesis conducente a la obtención del título de licenciado.

Puede aún identificarse una quinta etapa, que se inicia con la publicación de la Ley Universitaria 30220 el año 2014. La normativa complementaria posterior establece mecanismos de control de la calidad de las tesis, más rigurosos. Así, el Reglamento del Registro Nacional de Trabajos de Investigación para optar grados académicos y títulos profesionales-RENATI, (2016) dispone medidas de control para favorecer la originalidad de las tesis, sancionando el plagio. Podrá evaluarse la producción de tesis correspondientes a este período, después de transcurrido el quinto año de aplicación de las normas mencionadas.

La actividad académica universitaria incluye entre sus componentes la investigación científica con exigencias cada vez mayores, la Ley 30220 (Ley Universitaria), exige productos de investigación desde los más básicos niveles de formación académica, planteando así a los estudiantes y a las instituciones de educación superior, exigencias no consideradas en la ley anterior. El estudiante universitario y en especial el de La Escuela, tendrá que enfrentar estas exigencias y buscar, entre la producción científica almacenada, antecedentes que guíen su investigación. El presente estudio pretende aportar criterios orientadores de búsqueda tanto para los estudiantes de pre grado como para todo aquel estudioso que pretenda efectuar una revisión de las tesis almacenadas en La Escuela. Los objetivos del presente estudio son: cuantificar la producción de tesis, caracterizar su temática, analizar la fundamentación teórica desarrollada en cada una de ellas, analizar su planteamiento metodológico y realizar una prospección sobre la temática que se ha observado.

3 No se encuentra en la biblioteca, tesis producidas entre 1963 y 1971.

4 Se concluye la revisión de las tesis para el presente estudio, el 28 de noviembre del 2017. 


\section{MÉTODO}

\section{Población y muestra}

La población consta de 163 tesis en físico, que son todas las que se encuentran almacenadas en la biblioteca de La Escuela. Han sido excluidas para este efecto las tesinas que suman 89 , cuya estructura y condiciones de elaboración difieren de las que presentan las tesis. La muestra ha sido determinada con un nivel de confianza de $95 \%$ y un nivel de error de $8 \%$. De esta manera, el tamaño de la muestra es de 77 tesis. Cada una de las cinco etapas definidas desde la creación de La Escuela hasta la actualidad cuenta con una muestra específica que ha sido determinada mediante el procedimiento de muestreo estratificado con afijación proporcional al tamaño.

Los resultados más generales, han sido logrados analizando la lista de todas las tesis que figuran en el catálogo de la biblioteca. En la segunda parte, debido a las limitaciones temporales y logísticas presentes en este estudio, el análisis del contenido de cada tesis ha sido hecho recurriendo a la lectura de la muestra.

La estructura y contenido de las tesis producidas entre los años 1957 y 1974, no han permitido un análisis con los mismos criterios empleados para las épocas posteriores. Para este grupo de tesis se ha efectuado un análisis de contenido cualitativo breve, que difiere de los propósitos de un estudio bibliométrico. Se ha dado atención prioritaria a las características encontradas en las tesis desde 1994.

Hemos dado particular importancia al manejo de las citas dentro del marco teórico, para ello se optó por clasificar los tipos de citas con apoyo en la propuesta de Weinstock (1981, citado por Sabaj y Páez, 2011, pp. 122-123). Se ha considerado los tipos de citas que observamos más frecuentes a partir de una lectura preliminar de una muestra de las tesis motivo de estudio. A saber:

- Cita enunciativa, es decir aquella en la que se explicita el concepto hallado en una fuente. El tesista hace suyo el concepto que cita, pero sin comentarlo, analizarlo ni darle ningún tratamiento.

- Cita de corroboración, aquella en la que igualmente el tesista hace suyo el concepto que cita, pero seguido o antecedido por un análisis o comentario del tesista, de manera que la cita respalde lo afirmado por él.

- Cita de complementación, definida así aquella en la que se logra la elaboración de un sistema conceptual complejo, ordenando las citas de dos o más autores de manera que se complementen entre sí.

- Cita de comparación. Tienen el propósito de establecer semejanzas y diferencias entre las posiciones de dos o más autores. 


\section{RESULTADOS}

\section{Primeras tesis con las que cuenta La Escuela}

Primer período. En la biblioteca de la ENFJMA se ha encontrado un total de 11 tesis que pertenecen a esta etapa inicial de funcionamiento de La escuela que va de 1949 a 1964. La primera es del año 1957 y la última de este periodo corresponde a 1963. Estos trabajos se caracterizan por ser descripciones de fiestas costumbristas, danzas típicas y aspectos folklóricos de determinada comunidad. No tienen la estructura de una tesis propiamente dicha $y$, a pesar de presentar divisiones en capítulos, no se encuentra entre ellos diferenciaciones entre bases teóricas, metodología y resultados, caracterizándose por ser breves en extensión. En ellas se narran eventos concretos sobre la fiesta o danza, el lugar en que se desarrollan y características de los pobladores. Toda la redacción es propia, con gran ausencia de citas. Se observó poca presencia de referencias (bibliografía) y los trabajos que sí las contienen no presentan un formato definido por el cual se pueda determinar claramente la tipología documental de las fuentes. Los datos son obtenidos de hechos históricos, observación propia y de entrevistas a los pobladores del lugar. Algunos presentan ilustraciones, partituras y esquemas de bailes realizados a mano alzada.

Segundo periodo. Comprende 20 tesis, que van de 1965 a 1985. Se comienza a incluir el tema educativo en las tradiciones folklóricas sobre las que tratan, pero aún de forma muy discreta: sólo seis tesis presentan alguna aplicación específica a la Educación Básica Regular en general (EBR), o a sus niveles de Inicial o Primaria) ${ }^{5}$. La gran mayoría de los trabajos continúan en la línea de las manifestaciones costumbristas, aunque con orientaciones hacia conceptos pedagógicos y propuestas educativas.

La extensión de estos trabajos ya es mayor, se observa profundidad en la presentación del hecho folklórico con importantes hallazgos, pertinentes y relevantes. Aunque todavía no hay un correcto manejo de citas en las bases teóricas y en muchos casos se observa ausencia de las mismas. Por otro lado, la propuesta educativa carece de una fundamentación teórica sólida, carece de detalle en el contenido y en su secuenciación, lo que la hace deslucirse en su contrastación con la descripción del hecho folklórico que se constituye en la parte de más valor en el trabajo.

Estos trabajos incluyen fotografías, esquemas de bailes, diagramas y partituras. Muchos de ellos se elaboraron en base a la experiencia del investigador en el lugar de origen del hecho folklórico, en donde se entrevistó con los pobladores, quienes se constituyen en una de las principales fuentes de información y documentación. En estos trabajos se encuentra una mejor técnica en la presentación de las referencias bibliográficas, las que también se observan en mayor número que en la etapa anterior.

\section{Producción de tesis en números}

El total de tesis incluye 133 trabajos que han sido elaborados por un solo tesista,

5 El Ministerio de Educación de Perú denomina actualmente Educación Básica Regular al proceso de educación formal obligatorio, recibido en las escuelas por niños y jóvenes entre los 3 y los 17 años de edad. 
29 de las tesis corresponden a dos autores, existe una sola tesis elaborada por tres autores. Este grupo de coautoría se ha producido entre 1973 y 2014.

Se ha apreciado casi exclusividad femenina en la autoría de las tesis presentadas en los primeros años de La Escuela, ya que de las 31 tesis sustentadas entre 1949 y 1985, 29 fueron de mujeres y solo dos fueron presentadas por varones.

En total, el número de tesis de autoría femenina, duplica el número de tesis autoría masculina, sin embargo, si se observa específicamente la mención de música, el número de tesis de autoría masculina es seis veces el número de autoría femenina. Estas proporciones reflejan aproximadamente las proporciones que se observan actualmente en el sexo de los estudiantes matriculados en los últimos cinco años, aunque con crecimiento de la población de varones en las menciones de danza. No se ha podido obtener información de antaño sobre el particular. (Ver tabla 1).

Tabla 1

Cantidad de tesis según género, por mención

\begin{tabular}{lcccc}
\hline & Masculino & Femenino & Mixto & Total \\
\hline Danza & 18 & 73 & 7 & 98 \\
Música & 31 & 5 & 2 & 38 \\
Música y danza & 3 & 24 & 0 & 27 \\
Total & 52 & 102 & 9 & 163 \\
\hline
\end{tabular}

Durante los 61 años transcurridos a partir de la primera tesis con que cuenta la biblioteca de La Escuela, se observa fluctuaciones en la cantidad de tesis producidas por año, que no muestran una tendencia creciente o decreciente uniforme. Puede afirmarse que el período en el que se ha producido mayor cantidad de tesis es desde el año 2000 hasta el 2009. El año de mayor producción es el 2007, con un total de 18 tesis, mientras que no existe en la biblioteca ninguna tesis del 2013. No se ha determinado la causa de tales fluctuaciones. (Ver figura 1).

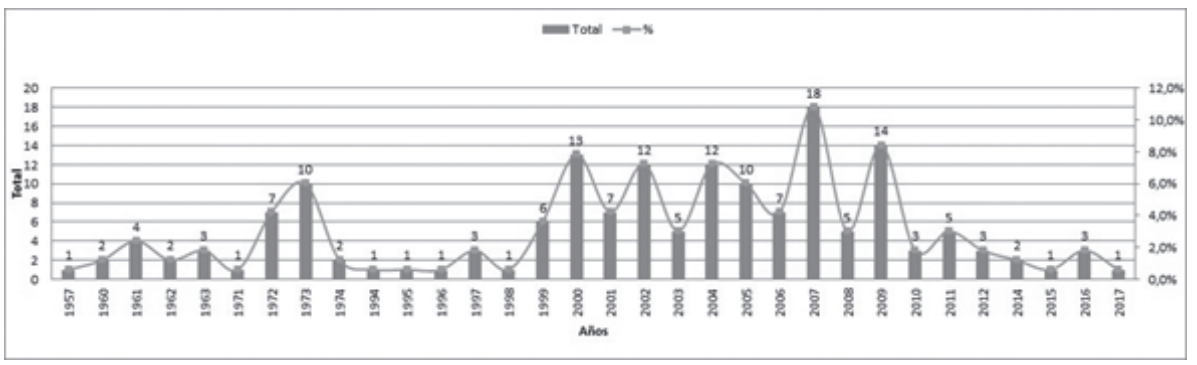

Figura 1. Producción de tesis por año.

\section{Caracterización temática}

En un $63 \%$ de las tesis, o sea 103 de ellas, puede observarse que la temática se inscribe dentro de una región política del Perú. En este conjunto de investigaciones, se privilegia la elección de la región Junín, como tema de investigación, seguida 
de Lima y en tercer lugar Ayacucho, con notable ventaja respecto de las demás regiones políticas. La cuantificación detallada se observa en la figura 2, pudiendo notarse que en sólo cuatro de las 25 regiones políticas del Perú se concentra más del $50 \%$ de las tesis con motivos regionales. Desde una óptica inversa, se hace patente la omisión de nueve regiones políticas, las cuales no son motivo de ninguna tesis. Toda la región natural selva ha quedado fuera del interés investigativo de los estudiantes. (Ver figuras 2 y 3 ).

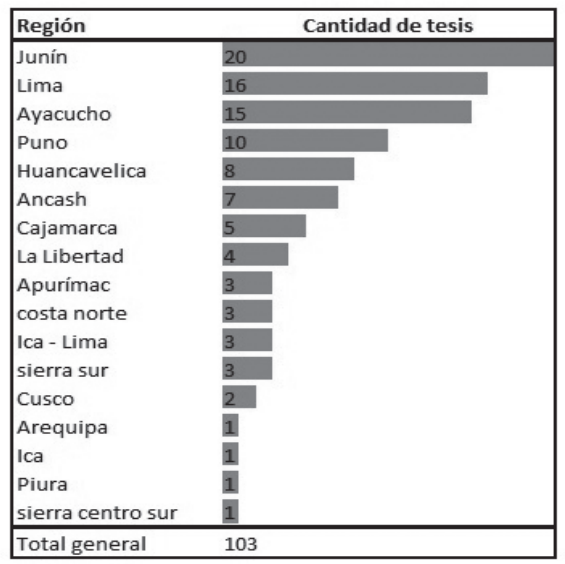

Figura 2. Regiones políticas consideradas en las tesis.

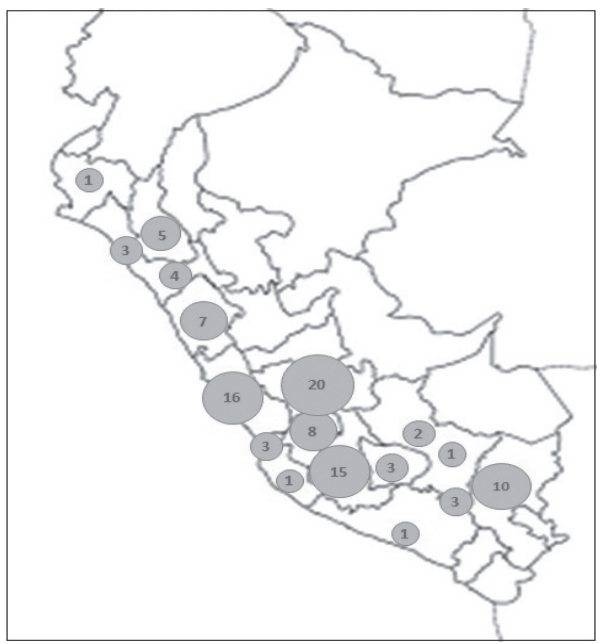

Figura 3. Distribución a nivel nacional de las tesis producidas. En esta imagen puede observarse 9 regiones políticas sobre las cuales no hay producción de trabajos de investigación; a saber: Loreto, Amazonas, San Martín, Huánuco, Pasco, Ucayali, Madre de Dios, Moquegua y Tacna. Siete de estas nueve regiones políticas ausentes en las tesis, pertenecen a la región natural Selva. 
En cuanto a las variables que son más recurrentes, figuran en primer lugar los temas que forman parte del concepto de identidad social, tales como: identidad nacional, identidad cultural, interculturalidad y otros, relacionados con las dificultades históricas de los peruanos para convivir en medio de diferencias culturales, históricas, económicas, geográficas; que devienen en la imposibilidad de arribar a una definición clara de la nación peruana. Tales trabajos suman un total de 21, conformando el $12,8 \%$ del total de las tesis. Sólo dos de estos trabajos pertenecen a estudiantes de la mención de Música, siento todos los demás de la mención de Danza. Este marcado interés en el tema se traduce en la realización de trabajos que se presentan como preexperimentos y proponen el "fortalecimiento", "reforzamiento" 0 "desarrollo" de la identidad mediante la enseñanza de una danza -generalmente andina- contenida en un programa de entre 8 y 14 sesiones de duración ${ }^{6}$, dirigido a grupos de estudiantes de un aula escolar dentro de Lima Metropolitana. Los resultados son medidos mediante una encuesta construida por el propio tesista. Se ha encontrado en todos los casos, elementos que invalidan o amenazan gravemente la validez de dicha encuesta.

Del conjunto de las tesis de la mención de Música, el 55\% están destinadas a demostrar la efectividad de la intervención pedagógica sobre el aprendizaje de un instrumento musical. Esto representa el siguiente $12,8 \%$ del total de las tesis que conforman la población de este estudio. (Ver tabla 2).

Tabla 2

Cantidad de tesis que tratan sobre la enseñanza de un instrumento musical.

\begin{tabular}{|lc|}
\hline Instrumentos & Total \\
Guitarra & 6 \\
Zampoña & 3 \\
Antara & 2 \\
Charango & 2 \\
Flauta & 2 \\
Quena & 2 \\
Batería & 1 \\
Siku & 1 \\
Quena y zampoña & 1 \\
Saxo y clarinete & 1 \\
Total General & 21 \\
\hline
\end{tabular}

En segundo lugar, por debajo de los dos temas anteriores, están las tesis orientadas a aspectos vinculados con el comportamiento motor, tales como la coordinación, el equilibrio, el esquema corporal, la lateralidad. Este grupo de tesis suma 16, conformando el 9,8\% del total de las tesis existentes. Se presenta el mismo diseño que en el caso de la identidad nacional, es decir, el preexperimento con el propósito de mejorar el aspecto del comportamiento motor, que se haya elegido. A pesar de que en las ciencias del comportamiento motor se cuenta con diversos instrumentos

6 Excepcionalmente se ha encontrado una tesis con un programa de 22 sesiones de aprendizaje y otra con 28 sesiones. 
de medición reconocidos por la literatura académica, se ha optado construir instrumentos con criterios diversos que no ha sido -excepto en una de las tesis revisadas- sometido a ningún procedimiento de validación. De un total de 16 tesis que conforman este grupo, 15 son de danza y una de música.

Respecto al ámbito al que se orientan las tesis, el $68 \%$ de ellas están dirigidas a temas correspondientes a la educación impartida en alguna entidad, sea ésta de naturaleza educativa artística u otra. Este grupo está inscrito en su mayoría dentro de la Educación Básica Regular, en el nivel primario; en segundo lugar en la Educación Básica Regular, nivel secundario. Es evidente el sesgo hacia el ámbito de la educación formal que se imparte a nivel escolar (Ver figura 4).

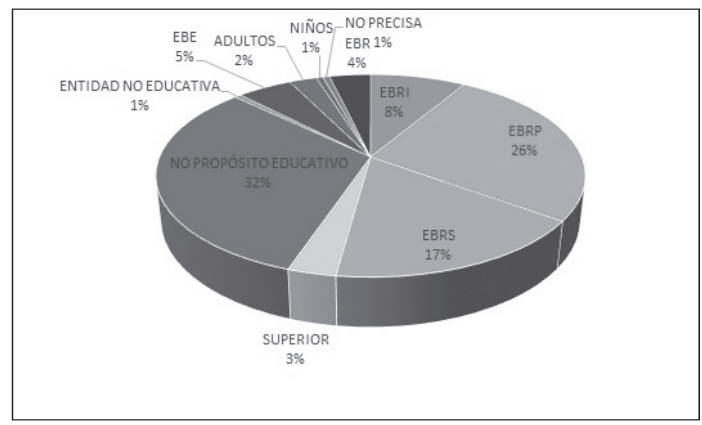

Figura 4. Niveles y modalidades educativas sobre los que se basaron los estudios: Educación Básica Regular (EBR), Educación Básica Regular Inicial (EBRI), Educación Básica Regular Primaria (EBRP), Educación Básica Regular Secundaria (EBRS), Educación Básica Especial (EBE), educación de adultos, educación de niños en general y educación en entidades no educativas.

\section{Análisis de las bases teóricas. ${ }^{7}$}

En cuanto a las citas. La cantidad total de citas contenidas en cada tesis es altamente fluctuante, desde dos tesis en las que no se encontró ninguna cita, hasta una de ellas que contiene un total de 235 citas. La mediana es 28 citas. En muchos casos no ha sido posible distinguir la cita textual de la paráfrasis, por lo que se ha contado todas bajo la definición general de "cita". La densidad de las citas (cantidad de citas por página), se expresa en el cociente de la cantidad de páginas entre la cantidad de citas. El mínimo hallado entre las tesis que presentan citas es una cita por cada 12 páginas y el máximo, una cita por cada 0.6 de página. La mediana es una cita por cada dos páginas. Debe considerarse que en muchas tesis las citas están concentradas en sólo una parte de las bases teóricas, mientras que existen numerosas páginas sin citas.

Se ha encontrado que la cita más empleada es la de tipo enunciativa, es decir aquella en la que se explicita el concepto hallado en una fuente, sin que el tesista analice

$7 \quad$ Todo este acápite ha sido elaborado contando con las tesis desde 1994 hasta el 2017, que suman un total de 132, de las cuales se ha extraído una muestra de 56, de acuerdo al procedimiento de muestreo estratificado con afijación proporcional al tamaño. 
o comente tal concepto. Se encuentra en el $100 \%$ de las tesis que presentan citas, desde 3 hasta 182 citas enunciativas por tesis.

En segundo lugar se encuentra la cita de corroboración, aquella que va precedida o seguida por un análisis o comentario del tesista. Se encuentra presente en el $36 \%$ de las tesis. La cantidad de este tipo de citas fluctúa entre cero y 82 por tesis. La observación nos permite afirmar provisionalmente que este tipo de cita se encuentra en mayor cantidad en los trabajos con mayor calidad argumentativa.

Las citas de complementación, definidas así aquellas en las que se logra la elaboración de un sistema conceptual con el aporte de dos o más autores citados, están presentes en el $23 \%$ de las tesis. Se ha hallado entre 1 y 6 citas de este tipo, por tesis.

Las citas con propósito de comparación de la posición de dos o más autores han sido halladas en el $11 \%$ de las tesis. En todos los casos el propósito ha sido establecer semejanzas entre distintos autores. No se ha encontrado citas empleadas para encontrar diferencias o contradicciones entre autores. La cantidad de citas de este tipo fluctúa entre 1 y 3 por tesis. (Ver tabla 3).

Tabla 3

Análisis del tipo de citas presente en las bases teóricas

\begin{tabular}{lrrrrrrrr}
\hline & $\begin{array}{r}\text { Cita } \\
\text { impertinente }\end{array}$ & $\begin{array}{r}\text { Cita } \\
\text { enunciativa }\end{array}$ & $\begin{array}{r}\text { Cita de } \\
\text { corroboración }\end{array}$ & $\begin{array}{c}\text { Citade } \\
\text { diferencia complementación }\end{array}$ & $\begin{array}{r}\text { Cita de } \\
\text { semejanza }\end{array}$ & $\begin{array}{r}\text { citade } \\
\text { juiciamiento }\end{array}$ \\
\hline Núm. Válido & 56.0 & 56.0 & 56.0 & 56.0 & 56.0 & 56.0 & 56.0 & 56.0 \\
Media & 0.2 & 36.3 & 5.1 & 0.0 & 0.5 & 0.1 & 0.0 & 8.3 \\
Mediana & 0.0 & 25.0 & 0.0 & 0.0 & 0.0 & 0.0 & 0.0 & 5.0 \\
Moda & 0.0 & $7^{*}$ & 0.0 & 0.0 & 0.0 & 0.0 & 0.0 & 0.0 \\
Desviación estándar & 1.1 & 38.8 & 13.7 & 0.0 & 1.2 & 0.5 & 0.3 & 10.0 \\
Rango & 8.0 & 182.0 & 82.0 & 0.0 & 6.0 & 3.0 & 2.0 & 44.0 \\
Mínimo & 0.0 & 0.0 & 0.0 & 0.0 & 0.0 & 0.0 & 0.0 & 0.0 \\
Máximo & 8.0 & 182.0 & 82.0 & 0.0 & 6.0 & 3.0 & 2.0 & 44.0 \\
\hline
\end{tabular}

a. Existen múltiples modos. Se muestra el valor más pequeño.

En cuanto a las fuentes. Se ha encontrado que la bibliografía utilizada tiene en su mayoría una antigüedad de 5 a 20 años; están en segundo lugar las fuentes con 5 o menos años de antigüedad, seguidas de aquellas con más de 20 años de antigüedad (Ver tabla 4).

Tabla 4

Antigüedad de la bibliografía empleada

\begin{tabular}{lrrrrr}
\hline & 5 años & $\begin{array}{r}\text { Entre } 5 y \\
20 \text { años }\end{array}$ & $\begin{array}{r}\text { Más de } \\
20 \text { años }\end{array}$ & $\begin{array}{r}\text { Bibliografía } \\
\text { sin fecha }\end{array}$ & $\begin{array}{r}\text { Cantidad de } \\
\text { documentos en } \\
\text { bibliografía }\end{array}$ \\
\hline Núm. Válido & 56.0 & 56.0 & 56.0 & 56.0 & 56.0 \\
Media & 6.5 & 16.3 & 5.1 & 5.7 & 33.7 \\
Mediana & 5.0 & 13.0 & 4.5 & 3.5 & 29.0 \\
Moda & 5.0 & $8 *$ & 2.0 & 0.0 & 29.0 \\
Desviación estándar & 7.3 & 11.9 & 4.2 & 6.9 & 22.8 \\
Rango & 36.0 & 62.0 & 22.0 & 38.0 & 112.0 \\
Mínimo & 0.0 & 0.0 & 0.0 & 0.0 & 0.0 \\
Máximo & 36.0 & 62.0 & 22.0 & 38.0 & 112.0 \\
\hline *Existen múltiples modos. Se muestra el valor más pequeño & & &
\end{tabular}


El tipo de documento más recurrido es claramente el libro, seguido del artículo publicado en revista científica. La cantidad de documentos que no ha sido posible tipificar, es alta (Ver tabla 5).

Tabla 5

Tipología documental empleada

\begin{tabular}{lrrrrrr}
\hline & Libro & Tesis & $\begin{array}{r}\text { Artículo en } \\
\text { revista } \\
\text { científica }\end{array}$ & $\begin{array}{r}\text { Documento } \\
\text { legal }\end{array}$ & $\begin{array}{r}\text { Documento } \\
\text { científico }\end{array}$ & Otros \\
\hline Núm. Válido & 56.0 & 56.0 & 56.0 & 56.0 & 56.0 & 56.0 \\
Media & 22.2 & 1.5 & 2.0 & 0.2 & 1.0 & 6.8 \\
Mediana & 19.0 & 0.0 & 0.0 & 0.0 & 0.0 & 4.5 \\
Moda & 16.0 & 0.0 & 0.0 & 0.0 & 0.0 & 0.0 \\
Desviación estándar & 13.9 & 2.4 & 4.5 & 0.7 & 2.2 & 7.9 \\
Rango & 71.0 & 9.0 & 23.0 & 3.0 & 12.0 & 32.0 \\
Mínimo & 0.0 & 0.0 & 0.0 & 0.0 & 0.0 & 0.0 \\
Máximo & 71.0 & 9.0 & 23.0 & 3.0 & 12.0 & 32.0 \\
\hline
\end{tabular}

\section{Análisis metodológico8}

En una visión integradora de las diferentes partes que constituyen una tesis, se ha procurado determinar la consistencia entre el título, marco teórico, hipótesis, resultados y conclusiones, encontrándose que la consistencia es una carencia evidente en la mayoría de trabajos analizados, tal como se puede observar en la figura 5.

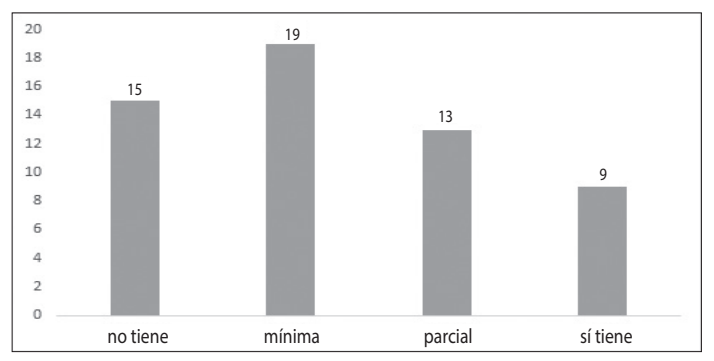

Figura 5. Consistencia entre título, objetivos, hipótesis y conclusiones.

No ha sido posible determinar ninguna tendencia temporal en cuanto a la consistencia, pues trabajos con diversos niveles de consistencia, se observan distribuidos sin ningún patrón evidente desde 1994 hasta el 2017. Es empero notable que, a partir del año 2010, no se produce ninguna tesis con aceptable consistencia, y que la situación se agrava a partir del año 2014, tal como se puede observar en la figura 6.

8 Al igual que en el acápite anterior, este ha sido elaborado contando con las tesis desde 1994 hasta el 2017, que suman un total de 132 , de las cuales se ha extraído una muestra de 56 , de acuerdo al procedimiento de muestreo estratificado con afijación proporcional al tamaño. 


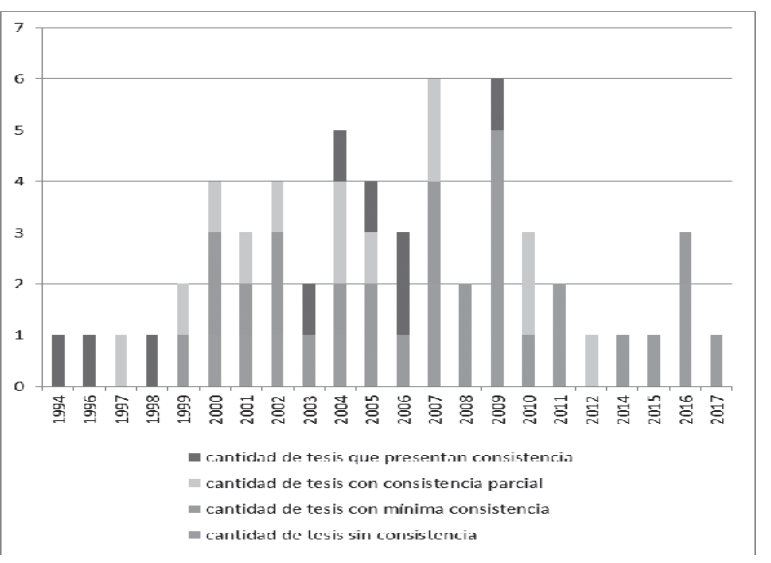

Figura 6. Comportamiento del nivel de consistencia, en el tiempo.

La extensión de los trabajos es muy heterogénea, variando principalmente por la existencia de "anexos" que pueden exceder el ciento de páginas. Se ha medido únicamente la extensión de las bases teóricas y de los resultados. ${ }^{9}$ Las bases teóricas fluctúan desde 11 páginas como mínimo hasta 245 como máximo, con una mediana de 68 páginas. En cuanto al capítulo de resultados, se ha determinado que presentan entre cero y 98 páginas, siendo la mediana 21. Realizada la comparación entre la extensión de los resultados y la extensión de las bases teóricas, se ha podido establecer que son mayoritarias las tesis con un marco teórico notablemente más voluminoso que el capítulo de resultados, tal como se puede apreciar en la tabla 6.

Tabla 6

Proporción entre resultados y bases teóricas

\begin{tabular}{ll}
\hline N. Válidos & 56 \\
\hline Media & 0.6 \\
Mediana & 0.3 \\
Moda & 0.5 \\
Desviación estándar & 1.2 \\
Rango & 8.2 \\
Mínimo & 0.0 \\
Máximo & 8.2 \\
\hline
\end{tabular}

Nota: Los valores indican el cociente de la división de resultados entre bases teóricas. Por ejemplo, siendo la mediana $(0,3)$, se puede decir que en este caso, por cada 10 páginas de bases teóricas, existirían tres páginas de resultados. En el caso del valor máximo $(8,2)$, por cada 10 páginas de bases teóricas, existirían 82 páginas de resultados.

9 La extensión ha sido medida en cantidad de páginas, habida cuenta de que se ha contado únicamente con la versión física de las tesis almacenadas en la biblioteca. 
Analizada esta proporción: resultados entre bases teóricas, en función del tiempo, podemos afirmar que las tesis con proporciones menores que 0,25 , es decir, aquellas en las cuales por cada 10 páginas de bases teóricas existen menos de 2,5 páginas de resultados, son un 37\% y se encuentran distribuidas desde el año 1999 hasta 2016. El siguiente $34 \%$ de tesis tienen una proporción hasta menos que 0,5 y se encuentran distribuidas, desde el año 1994 hasta el 2015. (Ver figura 7). No se observa ninguna tendencia en la distribución a lo largo del tiempo de existencia de La Escuela.

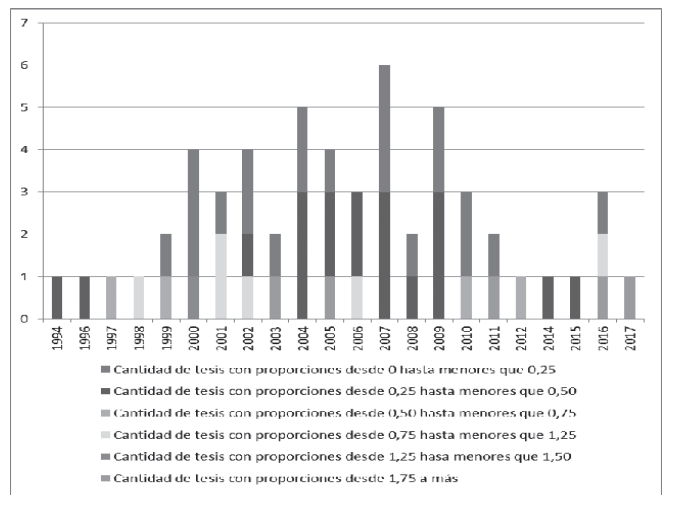

Figura 7. Proporción entre resultados y bases teóricas en función del tiempo.

En cuanto al proceso de validación de los instrumentos de acopio de datos, un $85 \%$ de aquellos que requieren algún procedimiento de validación, no lo tienen. El restante $15 \%$ cuenta con el procedimiento de juicio de expertos, adjuntando la firma de uno o más docentes en el documento respectivo, siempre en un mismo formato de redacción genérica. Los instrumentos así construidos evidencian deficiencias y carencias que se cuantifican en la figura 8.

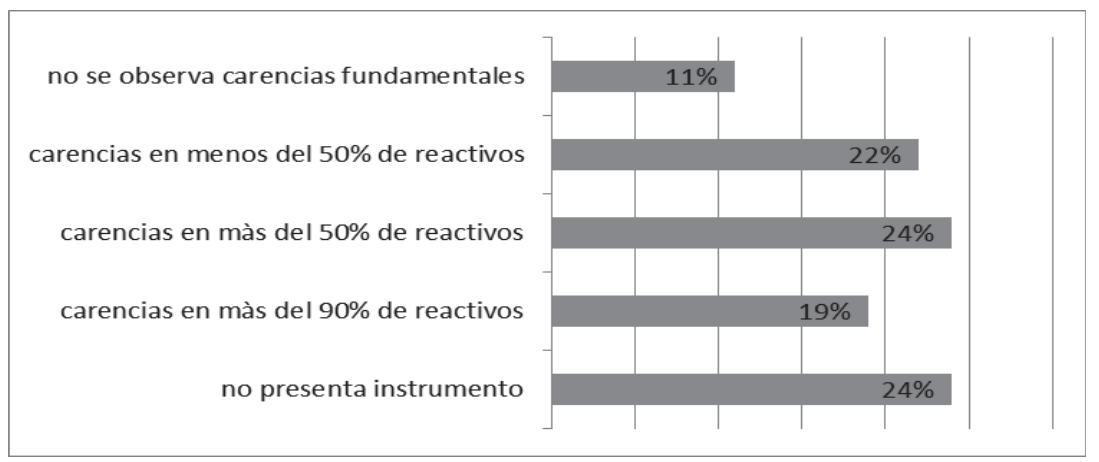

Figura 8. Indicadores de validez en los instrumentos de acopio de datos. Se ha considerado 47 tesis de la muestra, que son las que requieren de un instrumento de acopio de datos cuantitativos, validado. 
En cuanto a la validez de los instrumentos, tampoco ha sido posible encontrar una tendencia en el tiempo. Instrumentos con distintos niveles de validez se distribuyen desde el año 1997 hasta el 2017 sin ningún patrón evidente (Ver figura 9).

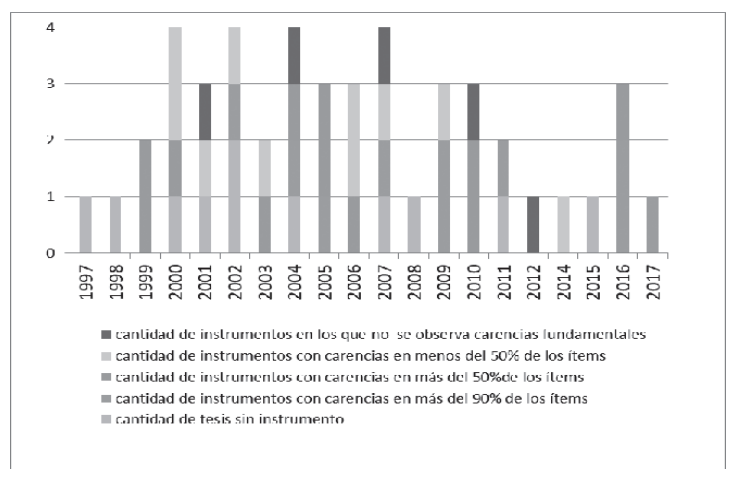

Figura 9. Validez del instrumento en función del tiempo.

Las tesis más leídas son 19 y han sido sustentadas entre 1998 y 2010 . El resto no son consultadas nunca o casi nunca. No se ha podido determinar alguna asociación entre las tesis más leídas y alguno de los rasgos de calidad en su elaboración. Existen tesis de egresados (entre 1996 y 2015), que han sido citadas en otras tesis, también de La Escuela; la tesis más citada, ha sido citada en otras seis tesis. Tampoco en este caso se logró encontrar asociación entre el número de veces que ha sido citada una tesis y la calidad en su elaboración. A su vez, no existe relación observable entre las tesis que han sido más citadas y las tesis que han sido más leídas.

En los casos en que se ha requerido el procedimiento de prueba de hipótesis, se ha encontrado invariablemente el uso de la T de Student como prueba de diferencia. Esta ha sido empleada sin discriminar si los datos tienen una distribución normal, tratándose de muestras pequeñas en la mayoría de casos. No obstante, al no ser posible conocer el tipo de distribución, hemos asumido que el procedimiento es correcto. En la mayoría de estas tesis no se ha recurrido a ninguna prueba estadística, solamente se ha hecho cálculos aritméticos básicos, lo que hemos considerado como inadecuado. (Ver figura 10).

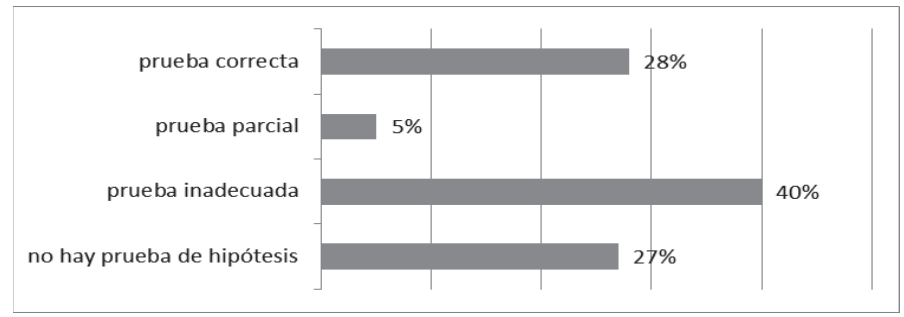

Figura 10. Prueba de hipótesis empleada. 
Es notorio que en los últimos 4 años no se ha encontrado dentro de la muestra, ninguna tesis en la que se haya manejado correctamente la prueba de hipótesis. En esta etapa: o no existe prueba de hipótesis, o la prueba es inadecuada. (Figura 11).

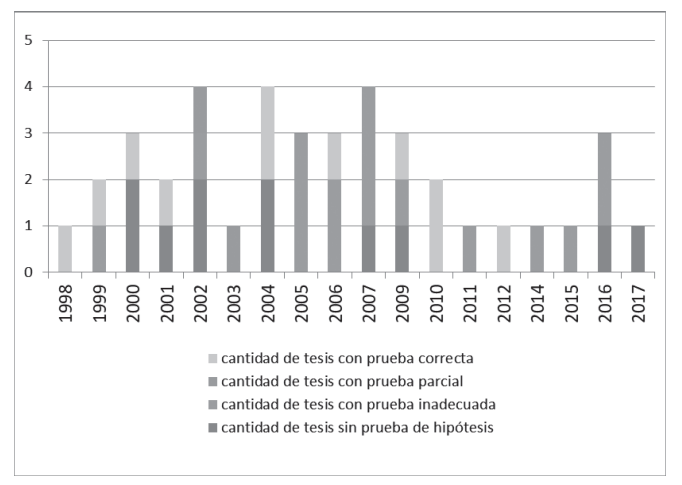

Figura 11. Prueba de hipótesis empleada, en función del tiempo.

\section{Prospección sobre la temática de las tesis}

Se ha efectuado una búsqueda con los datos de cada uno de los autores de las 163 tesis, en las bases datos Google y Google Académico, encontrándose mediante estas herramientas que son seis los autores que después de la elaboración de su tesis, han realizado un total de 12 publicaciones académicas ubicables en algún sitio web. Tales publicaciones son las que se observa en la tabla 7:

Tabla 7

Publicaciones halladas en sitios web

\begin{tabular}{|c|c|c|c|}
\hline Nombre & Año & Título de la publicación & Tipo \\
\hline Raffo Ze garra, Carmen Virginia & 1977 & LaActividad te atral escolar basada en elementos folklóricos & Tesis doctorado \\
\hline Carhuallanqui Berrocal, Rosa María & 1998 & Pastores de altura: magia, ritos y danza & Libro \\
\hline Molina Salce do, Javier Manuel & 2001 & Guitarra Pe ruana Se is cue rdas por los caminos del Pe rú Vol 1 & Libro \\
\hline Molina Salce do, Javier Manuel & 2003 & Guitarra Peruana Se is cuerdas por los caminos del Perú Vol 2 & Libro \\
\hline Molina Salce do, Javier Manuel & 2005 & Guitarra Pe ruana Se is cue rdas por los caminos del Perú Vol 3 & Libro \\
\hline Molina Salce do, Javier Manuel & 2007 & Guitarra Peruana Se is cue rdas por los caminos del Pe rú Vol 4 & Libro \\
\hline Pérez Sandoval, Johnny Marino & 2008 & Aprendiendo a tocar música & Libro \\
\hline Molina Salce do, Javier Manuel & 2009 & Guitarra Peruana Música populartradicional Vol 1 & Libro \\
\hline Molina Salce do, Javier Manuel & 2009 & Guitarra Peruana Música populart radicional Vol 2 & Libro \\
\hline Molina Salce do, Javier Manuel & 2009 & Guitarra Peruana Se is cuerdas por los caminos del Pe rú Vol 5 & Libro \\
\hline Rojas Vásquez, Policarpio Saúl & 2014 & Teoría de ladanzatradicional & Libro \\
\hline Alvarez Aliaga, Alex & 2017 & Core ografía de la danza peruana de la tradición al espectáculo contemporáneo & Libro \\
\hline
\end{tabular}

En cuanto a los intereses de los actuales estudiantes de La Escuela, que a la fecha cursan el penúltimo o último año académico de estudios, se puede establecer en base a una encuesta efectuada en este grupo, que es predominante la elaboración 
de proyectos de investigación en los que se propone la modificación de alguna característica o rasgo de los educandos, merced a la intervención pedagógica del tesista.

Un 85\% de los estudiantes considera que entre los factores que vienen afectando negativamente sus esfuerzos para desarrollar la tesis, se encuentran (a) la falta de orientación y apoyo de los docentes, (b) las contradicciones entre los mismos respecto a los proyectos en elaboración, y (c) la negativa de la entidad ante determinados temas de investigación propuestos por los alumnos.

Obra en poder de los estudiantes el documento: Pautas para la elaboración de proyectos de tesis de pre grado [sic], en el que se regula y limita la elección de los temas de investigación mediante la siguiente disposición "La investigación en educación artística y folklore se debe desarrollar en el contexto de la educación formal (educación básica regular, educación básica alternativa, educación básica especial y educación superior)" (p. 4).

\section{DISCUSIÓN}

La construcción de la tesis se ha efectuado en muchos casos dentro de esquemas y formatos incompatibles con la naturaleza de la tesis elaborada, por lo cual no se ha podido evaluar en su debida dimensión algunos trabajos hechos con rigor lógico y evidente esfuerzo intelectual. Así, algunos marcos teóricos con evidentes indicadores de buena elaboración, son el preámbulo de un capítulo de resultados irrelevante o inconsistente. Del mismo modo, se ha observado resultados que indican una esforzada y competente elaboración, acompañados de marcos teóricos muy frágiles. También se ha encontrado párrafos o páginas completas, ubicados fuera del acápite adecuado; en tales casos se ha procedido en correspondencia con los encabezados utilizados por el autor de la tesis, lo que ha obligado a desestimar alguna información ubicada fuera de lugar.

Hemos determinado la validez de los instrumentos de acopio de datos únicamente en base a criterios técnicos y metodológicos generales para la construcción de instrumentos en la investigación científica. No se ha revisado el contenido del instrumento en función de la especialidad, por requerirse para ello un equipo de investigación interdisciplinario.

Se ha constatado un frecuente uso del superíndice en los encabezados dentro de las bases teóricas, que lleva a un pie de página en el que se consigna una fuente, sin más explicación, como si todo ese acápite hubiera sido extraído de aquella fuente. Esto no ha permitido identificar con precisión la extensión de las citas.

Los resultados referidos al tratamiento de la identidad social en las tesis analizadas, contrastados con los estudios de Giménez (1992); Espinosa (2003); Scandroglio, López y San José (2008), permiten concluir que el tratamiento teórico adolece de excesiva simplificación, pues el concepto de identidad social en cualquiera de sus manifestaciones (nacional, regional u otra) es de por sí complejo. Los resultados, 
mostrados en tales tesis, contrastados con lo sostenido por Fuller (2002), Herrera, Pinilla y Suaza (2003), sugieren que el proceso de socialización conducente a la formación de la identidad en el educando, no es esencialmente modificable mediante la enseñanza de una danza folklórica durante no más de 14 sesiones de aprendizaje, con las limitaciones de un trabajo preexperimental de pregrado. Por tanto, los propósitos de las tesis de tal naturaleza deben reajustarse.

La temática que se ha identificado como más atendida en las tesis, así como los temas que han sido objeto de desatención por parte de los estudiantes, tienen correlato en fenómenos culturales, histórico-sociales (Andrade, 2009); y económicos (INEl, 2017), que muestran un desbalance y desigualdad análogos, en la dinámica de las distintas regiones y estratos de la sociedad peruana. Es decir, que la misma desatención como objeto de estudio de tesis, observada con respecto a la región natural selva se presenta como desatención en términos de sociedad excluida.

En muchos casos, no se ha podido diferenciar las citas directas de las citas indirectas, por existir errores técnicos en la redacción, que dificultaron o imposibilitaron la tarea.

Existen en el campo de la bibliometría, recursos como el concepto de vida media de una fuente. Para la determinación de la vida media es empleado el índice de Price en estudios como el de Ortiz (2012), mediante el cual se determina la obsolescencia de las referencias, estableciéndose un patrón de cinco años de antigüedad. El presente estudio no ha tomado esos valores para el análisis de las fuentes puesto que la investigación en folklore puede hacer valiosa y en muchos casos imprescindible la consideración de fuentes más o menos antiguas.

\section{REFERENCIAS}

Andrade, L. y Pérez, J. (2009). Las lenguas del Perú. Lima: Pontificia Universidad Católica del Perú.

Arana, V. H. (2007). Historia Institucional. Lima: Escuela Nacional Superior de Folklore José María Arguedas.

Escuela Nacional Superior de Folklore José María Arguedas (2017). Pautas para la elaboración de proyectos de tesis de pre grado.

Espinosa, A. (2003). Identidad social e identidad nacional en una muestra de triciclistas de Juliaca. (Tesis de licenciatura). Pontificia Universidad Católica del Perú. Recuperado de http://bit.ly/2kbNBN6

Fuller, N. (2002). Masculinidades. Cambios y permanencias. Lima: Universidad Católica del Perú.

Giménez, G. (1992). La identidad social o el retorno del sujeto en sociología.Versión (2), 183-205. Recuperado de: http://bit.ly/2zCoPZw 
Herrera, M. C., Pinilla, A. V. y Suaza, L. M. (2003). La identidad nacional en los textos escolares de ciencias sociales. Colombia: 1900-1950. Bogotá: Universidad Pedagógica Nacional.

INEI (2017). Perú perfil de la pobreza por dominios geográficos 2007-2016. Lima: Autor.

Ley № 29292. (2008). Ley que modifica el artículo $1^{\circ}$ de la Ley № 26251, modificada por la Ley № 26341, que modifica el artículo $99^{\circ}$ de la Ley № 23733, Ley Universitaria.

Ley № 30220. (2014). Ley Universitaria.

Ortiz, S. (2012). Perfil de las tesis doctorales presentadas en el Departamento de Biblioteconomía y Documentación, de la Universidad Carlos III, durante los años académicos 1994/95- 2005/06. (Tesis doctoral). Universidad Carlos III. Madrid. Recuperada de http://bit.ly/2in0jlx

Reglamento del Registro Nacional de Trabajos de Investigación para optar grados académicos y títulos profesionales - RENATI - aprobado por Resolución del Consejo Directivo №. 033 - 2016 - SUNEDU/CD.

Scandroglio, B., López, J. S. y San José, M. C. (2008). La Teoría de la Identidad Social: una síntesis crítica de sus fundamentos, evidencias y controversias. Psicothema 20 (1), 80-89. Recuperado de: http://www.redalyc.org/html/727/72720112/

Sabaj, O. y Páez, D. (2011). Tipos y funciones de las citas en artículos de investigación de tres disciplinas. Literatura y lingüística, (22), 117-134. Recuperado de https:// scielo.conicyt.cl/pdf/lyl/n22/art09.pdf 